\title{
Impulse Noise Removal using Optimal Direction Method with Fuzzy based Median Filter
}

\author{
Raja S \\ Christ the King Engineering \\ College \\ Karamadai, Coimbatore, \\ Tamilnadu, India
}

\author{
C.Suresh Gnana Dhas, \\ Ph.D \\ Vivekanandha College of \\ Engineering for Women \\ Thiruchencode \\ Tamilnadu, India
}

\author{
V.Suresh babu \\ Christ the King Engineering \\ College \\ Karamadai, Coimbatore, \\ Tamilnadu, India
}

\begin{abstract}
Impulse noise is a spark that affects the contents of digital images. The proposed method detects the random valued impulse noise efficiently. Then the detected noisy pixels are restored by the median of neighbouring noise-free pixels. In a detection window of this proposed method, four directions are considered. From that, optimal direction is obtained by standard deviation. Threshold value is calculated by finding normalised distance between original pixel and other pixels in the optimal direction. The threshold value is used as a measure to detect whether the tested pixel is noisy or noise-free pixel. More edge pixels can be detected if the accurate or optimal direction of the edge is determined. The noisy pixel that has small deviations with the pixels in the optimal direction is seems like the original pixel. Here, in detection and in filtering, the window size is adaptive which depends on noise density in the detection window frame. The optimum threshold limit is fixed as 0.8 by using normalised distance between the central pixel and pixels in the optimum direction in first iteration. In second iteration, the threshold value should be kept very close to the value zero to remove the undetected noisy pixel and that was found as 0.4. In second iteration the noisy pixels will be mostly eliminated. It is found that the proposed method gives better results when compared to adaptive median filter, progressive switching median filter, in terms of PSNR and MSE values and output images are compared using MATLAB.
\end{abstract}

\section{General Terms}

Noise Removal, optimal direction

\section{Keywords}

Optimal direction, PSNR, MSE, MATLAB

\section{INTRODUCTION}

Digital images acquired through many consumer electronic products are commonly subjected to the contamination of impulse noise. In the process of image acquisition and transmission, impulse noises often cause serious degradation of the image quality. Images are frequently corrupted by impulse noise due to camera sensors or transmission in noisy channels [1]. It occurs in bioluminescence imaging for which the image acquisition is disturbed by the presence of cosmic noise. Cosmic noise can be considered to be an impulse noise. For this application in bioluminescence imaging, a step of filtering is needed as a pre-processing step before the deconvolution task.
Impulse noise is often introduced into images during acquisition and transmission. Based on the noise values, it can be classified as the easier-to-restore salt-and-pepper noise and the more difficult random-valued impulse noise [1]. There have been much more methods for removing the former and some of them have performed very well [2][5]. So here, we only focus on removing the latter. Among all kinds of methods for impulse noise, the median filter [6] is used widely because of its effective noise suppression capability and high computational efficiency. However, it uniformly replaces the gray-level value of every pixel by the median of its neighbors. Consequently, some desirable details are also removed, especially when the window size is large. In order to improve the median filter, many filters with an impulse detector are proposed, such as signal-dependent rank order mean (SD-ROM) filter [7], multistate median (MSM) filter [1],adaptive center weighted median (ACWM) filter [8], the pixel-wise MAD (PWMAD) filter [9], and iterative median filter [10].These filters usually perform well, but as the noise level is higher than $30 \%$, they tend to remove many features from the images or retain too much impulse noise. Recently, robust statistics based filters have emerged for the removal of impulse noise. The most recent statistic is rank ordered-relative difference (RORD) [9], which is used to detect more edge pixels, by implementing the wellknown statistic of rank-ordered absolute difference (ROAD) [10] not on the noisy image, but on the relative difference image. Though RORD delivers decent results, it still exhibits bad performance, in the case of blotches of noise, where the intensity differences between the neighboring pixels are small.

In this paper, we propose a novel algorithm based on finding the optimal direction used as a measure to detect whether the tested pixel is noisy or noise-free pixel. More edge pixels can be detected if the accurate or optimal direction of the edge is determined. The noisy pixel that has small deviations with the pixels in the optimal direction is deemed an original pixel. This letter is organized, after the introduction, as follows. In Section 2, we explain the proposed algorithm. In Section 3, we demonstrate numerical results and visual examples from simulations, and finally, we give a conclusion in Section 4.

\section{PROPOSED METHOD}

In algorithm description method, the filtering window size is considered as odd integer (e.g., 9). Then four directions of the filtering window is considered as four vector i.e., two diagonals and a vertical column and a horizontal row which passes through the center pixel. In this, optimal 
direction should be found using standard deviation to calculate a parameter ('s' here) which should be compared with the threshold limit to detect where the centre pixel is noisy or noiseless. The algorithm steps as follows:

- $\quad$ STEP 1: Assigning window size as fixed as $\mathrm{k}$. considering four direction in the window, then arranging those (k-1)pixel positions.

$D_{1}^{0,0}=\{(0,0),(1,1),(2,2),(3,3),(5,5),(6,6),(7,7),(8,8)\}$

$\mathrm{D}_{2}^{0,0}=\{(0,8),(1,7),(2,6),(3,5),(5,3),(6,2),(7,1),(8,0)\}$

$\mathrm{D}_{3}^{0,0}=\{(0,4),(1,4),(2,4),(3,4),(5,4),(6,4),(7,4),(8,4)\}$

$\mathrm{D}_{3}^{0,0}=\{(4,0),(4,1),(4,2),(4,3),(4,5),(4,6),(4,7),(4,8)\}$

- STEP 2: Then those pixel values should be sorted ascendingly,

$\mathbf{r}_{\mathrm{d}}^{\mathrm{ij}}=\left\{x_{1, s}^{d} \mid x_{1, s}^{d} \in D_{d}^{i j}, s=1: k-1, d=1: 4, x_{1, s+1} \geq x_{1, s}\right\}$

- STEP 3: Outlier pixels are removed, then to choose optimal direction, standard deviation of those pixel values of four direction is compared. The direction which gives minimum standard deviation is considered as optimum.

$$
\widetilde{\boldsymbol{r}}_{d}^{i j}=\left\{\boldsymbol{x}_{1, s}^{d} \mid s=2: k-2, d=1: 4\right\}
$$

- $\quad$ STEP 4:Selection of optimal direction by taking minimum standard deviation $\sigma$ :

$$
D^{o p}=\underset{\widetilde{r}_{d}^{i j}}{\arg \min }\left\{\sigma_{\widetilde{r}_{d}^{i j}}\right\}
$$

- $\quad$ STEP 5: Parameter $S$ is calculated using pixel values in the optimal direction, $\mathrm{S}$ is defined as

$$
S=\sum_{s=2}^{k-2}\left|\frac{x_{1, s}^{o p}-x_{\widetilde{i j}}}{255}\right|
$$

where $\mathcal{X}_{1, s}^{o p}$ is pixel s in the optimal direction.

- $\quad$ STEP 6: Threshold is set to define noisy and noiseless pixel.

- $\quad$ STEP 7: Then in filtering section, the noisy pixels are eliminated in the filtering window frame which is initially considered as $3 * 3$. If all the pixels in the frame is noisy, then the window size is increased so that we can get at least one noise free pixel in the frame.

- $\quad$ STEP 8: Then the median of noise free pixels of the frame is eliminated.

- $\quad$ STEP 9: for high noise density, if necessary we can go for second iteration.

\section{RESULTS AND COMPARISIONS}

Table 1. Comparison of PSNR values of proposed technique with existing techniques for $512 \times 512$ Baboon image

\begin{tabular}{|l|l|l|l|l|}
\hline $\begin{array}{l}\text { Noise } \\
\text { Density( } \\
\text { \%) }\end{array}$ & $\begin{array}{l}\text { PWMA } \\
\text { D }\end{array}$ & TSM & PSMF & $\begin{array}{l}\text { Propose } \\
\text { d filter }\end{array}$ \\
\hline 10 & 23.6 & 16.64 & 23.73 & $\mathbf{2 3 . 6 8}$ \\
\hline 20 & 23.51 & 16.22 & 22.68 & $\mathbf{2 3 . 1 8}$ \\
\hline 30 & 20.93 & 16.09 & 21.77 & $\mathbf{2 2 . 7 2}$ \\
\hline 40 & 18.67 & 16.12 & 20.95 & $\mathbf{2 1 . 7 3}$ \\
\hline 50 & 16.48 & 16.12 & 20.16 & $\mathbf{2 1 . 9 9}$ \\
\hline 60 & 14.59 & 16.16 & 19.14 & $\mathbf{2 0 . 7 9}$ \\
\hline 70 & 13.13 & 16.22 & 17.48 & $\mathbf{2 0 . 2 5}$ \\
\hline 80 & 11.92 & 16.18 & 15.25 & $\mathbf{1 9 . 1 2}$ \\
\hline 90 & 10.91 & 16.22 & 13.54 & $\mathbf{1 7 . 1 4}$ \\
\hline
\end{tabular}

Table 2. Comparison of MSE values of proposed technique with existing techniques for $512 \times 512$ Baboon image

\begin{tabular}{|l|l|l|l|l|}
\hline $\begin{array}{c}\text { Noise } \\
\text { Density(\% } \\
\text { ) }\end{array}$ & $\begin{array}{c}\text { PWMA } \\
\text { D }\end{array}$ & TSM & PSMF & $\begin{array}{c}\text { Propose } \\
\text { d filter }\end{array}$ \\
\hline 10 & 138.42 & $\begin{array}{l}963.1 \\
2\end{array}$ & 160.4 & 134.12 \\
\hline 20 & 221.5 & $\begin{array}{l}942.1 \\
2\end{array}$ & $\begin{array}{l}253.1 \\
8\end{array}$ & 241.35 \\
\hline 30 & 553.9 & $\begin{array}{l}912.5 \\
2\end{array}$ & $\begin{array}{l}329.7 \\
3\end{array}$ & 421.65 \\
\hline 40 & 580.45 & $\begin{array}{l}902.1 \\
5\end{array}$ & $\begin{array}{l}486.7 \\
8\end{array}$ & 550.11 \\
\hline 50 & 647.12 & $\begin{array}{l}902.1 \\
5\end{array}$ & 632.6 & 589.56 \\
\hline 60 & 800.70 & $\begin{array}{l}922.2 \\
6\end{array}$ & $\begin{array}{l}945.8 \\
1\end{array}$ & 602.54 \\
\hline 70 & 1285.63 & 942.1 & $\begin{array}{l}1352 . \\
4\end{array}$ & 688.18 \\
\hline 80 & 2986.36 & $\begin{array}{l}938.6 \\
3\end{array}$ & $\begin{array}{l}2573 . \\
8\end{array}$ & 888.56 \\
\hline 90 & 4587.36 & 942.1 & 3728. & 1356.23 \\
& & 2 & 4 & \\
\hline
\end{tabular}

Table 3. Comparison of SSIM values of proposed technique with existing techniques for $512 \times 512$ Baboon image

\begin{tabular}{|l|l|l|l|l|}
\hline $\begin{array}{c}\text { Noise } \\
\text { Density(\%) }\end{array}$ & PWMAD & TSM & PSMF & $\begin{array}{c}\text { Proposed } \\
\text { filter }\end{array}$ \\
\hline 10 & 0.85 & 0.76 & 0.95 & 0.96 \\
\hline 20 & 0.82 & 0.75 & 0.85 & 0.95 \\
\hline 30 & 0.76 & 0.72 & 0.82 & 0.93 \\
\hline 40 & 0.68 & 0.72 & 0.77 & 0.91 \\
\hline 50 & 0.62 & 0.75 & 0.68 & 0.85 \\
\hline 60 & 0.52 & 0.73 & 0.54 & 0.81 \\
\hline 70 & 0.56 & 0.68 & 0.40 & 0.74 \\
\hline 80 & 0.48 & 0.71 & 0.38 & 0.72 \\
\hline 90 & 0.35 & 0.65 & 0.29 & 0.68 \\
\hline
\end{tabular}


Table 4. Comparison of PSNR values of proposed technique with existing techniques for $512 \times 512$ Bridge image

\begin{tabular}{|c|c|c|c|c|}
\hline $\begin{array}{c}\text { Noise } \\
\text { Density(\%) }\end{array}$ & PWMAD & TSM & PSMF & $\begin{array}{c}\text { Proposed } \\
\text { filter }\end{array}$ \\
\hline 10 & 24.21 & 17.48 & 26.42 & 26.56 \\
\hline 20 & 23.57 & 16.66 & 25.56 & 25.75 \\
\hline 30 & 21.13 & 16.62 & 23.72 & 24.82 \\
\hline 40 & 18.60 & 16.71 & 22.21 & 23.63 \\
\hline 50 & 16.49 & 16.61 & 20.93 & 22.44 \\
\hline 60 & 14.64 & 16.62 & 19.37 & 21.22 \\
\hline 70 & 13.14 & 16.63 & 17.83 & 19.66 \\
\hline 80 & 11.93 & 16.68 & 15.68 & 17.94 \\
\hline 90 & 10.98 & 16.65 & 13.78 & 15.21 \\
\hline
\end{tabular}

Table 5. Comparison of MSE values of proposed technique with existing techniques for $512 \times 512$ Bridge image

\begin{tabular}{|c|c|c|c|c|}
\hline $\begin{array}{c}\text { Noise } \\
\text { Density(\%) }\end{array}$ & $\begin{array}{c}\text { PWMA } \\
\text { D }\end{array}$ & TSM & PSMF & $\begin{array}{c}\text { Propose } \\
\text { d filter }\end{array}$ \\
\hline 10 & 201.9 & 811.6 & 124.98 & 130.381 \\
\hline 20 & 261.22 & 924.5 & 151.31 & 157.32 \\
\hline 30 & 504.41 & 934.67 & 191.87 & 201.9 \\
\hline 40 & 802.15 & 923.5 & 404.31 & 268.22 \\
\hline 50 & 985.63 & 932.5 & 843.89 & 340.22 \\
\hline 60 & 999.23 & 912.8 & 1121.9 & 511.43 \\
\hline 70 & 1025.12 & 922.65 & 1794.4 & 622.76 \\
\hline 80 & 1500.68 & 923.38 & 2498.5 & 840.75 \\
\hline 90 & 2986.87 & 922.6 & 2883.6 & 1050.01 \\
\hline
\end{tabular}

Table 6. Comparison of SSIM values of proposed technique with existing techniques for $512 \times 512$ Bridge image

\begin{tabular}{|c|c|c|c|c|}
\hline $\begin{array}{c}\text { Noise } \\
\text { Density(\%) }\end{array}$ & PWMAD & TSM & PSMF & $\begin{array}{c}\text { Proposed } \\
\text { filter }\end{array}$ \\
\hline 10 & 0.87 & 0.86 & 0.96 & 0.98 \\
\hline 20 & 0.82 & 0.82 & 0.86 & 0.97 \\
\hline 30 & 0.78 & 0.72 & 0.82 & 0.96 \\
\hline 40 & 0.68 & 0.70 & 0.81 & 0.95 \\
\hline 50 & 0.66 & 0.68 & 0.75 & 0.88 \\
\hline 60 & 0.58 & 0.62 & 0.54 & 0.83 \\
\hline 70 & 0.48 & 0.58 & 0.39 & 0.78 \\
\hline 80 & 0.38 & 0.52 & 0.33 & 0.72 \\
\hline 90 & 0.25 & 0.35 & 0.25 & 0.65 \\
\hline
\end{tabular}

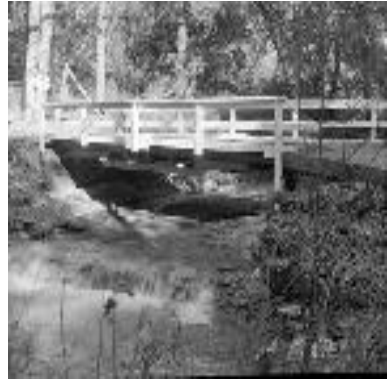

(a)

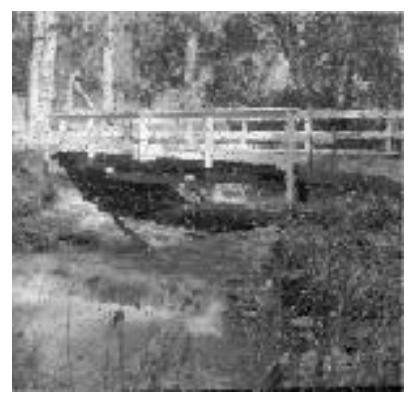

(c)

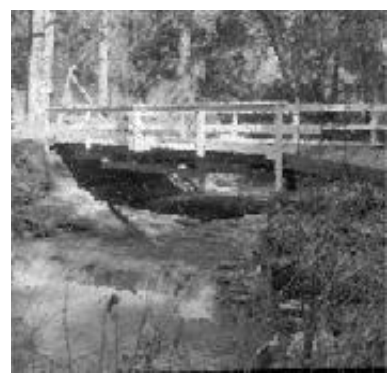

(e)

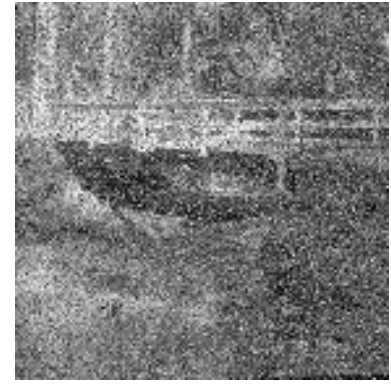

(b)

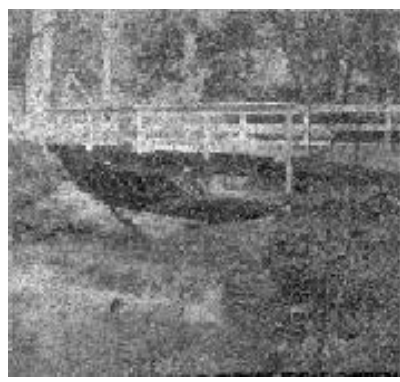

(d)

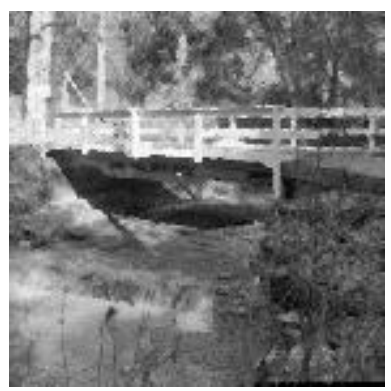

(f)
Fig.1. Denoising Using different Algorithms for bridge Image With Noise density of $40 \%$ a) Original Image b) Noisy Image c) Output of SMF d)Output of AMF e) Output of PSMF f) Output of proposed

\section{CONCLUSION}

In this paper, problem of image denoising was considered. Particular attention was paid to random valued impulse noise removal. The proposed approach is based on finding the optimal direction that is considered as a measure to indicate whether the tested pixel is noisy or noise-free pixel. In the sense that any pixel has small deviations with the pixels in the optimal direction is deemed as an original pixel. When compared to other algorithms for random valued impulse noise, proposed algorithm gives high performance for high noise densities. Because of complicated arithmetic and logical functions in each and every loop, the run time of this algorithm will be high. In future, this can be avoided using simple logical technique. 


\section{ACKNOWLEDGMENT}

Our sincere thanks to Dr.S.N.Sivanandam, Professor, Department of CSE, Karpagam College of Engineering, Coimbatore, Tamilnadu for his valuable guidance

\section{REFERENCES}

[1] T. Chen and H. R.Wu, "Space variant median filters for the restoration of impulse noise corrupted images," IEEE Trans. Circuits Syst. II, vol. 48, no. 8, pp. 784-789, Aug. 2001.

[2] H. Hwang and R. A. Haddad, "Adaptive median filters: new algorithms and results," IEEE Trans. Image Process., vol. 4, no. 4, pp. 499-502, Apr. 1995.

[3] S. Zhang and M. A. Karim, "A new impulse detector for switching median filters," IEEE Signal Process. Lett., vol. 9, no. 11, pp. 360-363,Nov. 2002.

[4] R. H. Chan, C.-W. Ho, and M. Nikolova, "Salt-andpepper noise removal by median-type noise detectors and detail-preserving regularization," IEEE Trans. Image Process., vol. 14, no. 10, pp. 1479-1485,Oct. 2005.

[5] P.-E. Ng and K.-K. Ma, "A switching median filter with boundary discriminative noise detection for extremely corrupted images," IEEE Trans. Image Process., vol. 15, no. 6, pp. 1506-1516, Jun. 2006.

[6] W. K. Pratt, "Median filtering," Image Process. Inst., Univ. Southern California, Los Angeles, Sep. 1975, Tech. Rep.

[7] E. Abreu, M. Lightstone, S. K. Mitra, and K. Arakawa, "A new efficient pproach for the removal of impulse noise from highly corrupted images," IEEE Trans. Image Process., vol. 5, no. 6, pp. 1012-1025, Jun. 1996.

[8] T. Chen and H. R. Wu, "Adaptive impulse detection using center-weighted median filters," IEEE Signal Process. Lett., vol. 8, no. 1, pp. 1-3, Jan. 2001.

[9] H. Yu, L. Zhao, and H. Wang, "An efficient procedure for removing random-Valued impulse noise in images," IEEE Signal Process. Lett.,vol. 15, no. 1, pp. 922-925, Dec. 22, 2008.

[10] R. Garnett, T. Huegerich, C. Chui, and W.-J. He, “A universal noise removal algorithm with an impulse detector," IEEE Trans. Image Process., vol. 14, no. 11, pp. 1747-1754, Nov. 2005. 